\title{
Análisis de la interacción de maestros cuando resuelven problemas realistas conjuntamente con sus alumnos en aulas de primaria, teniendo en cuenta su experiencia docente
}

Analysis of the interaction of teachers when they solve realistic problems along with their students in primary classrooms, taking into account their teaching experience

\section{Beatriz Sánchez-Barbero*}

(iD) : https://orcid.org/0000-0001-9118-2118

$$
\text { María Calatayud }{ }^{* *}
$$

(iD) : https://orcid.org/0000-0002-3799-629X

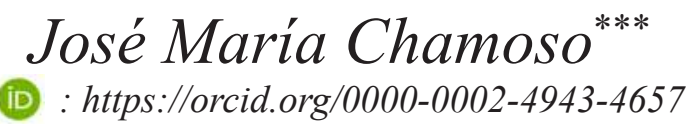

Tipo de Artículo: Informes de Investigación y ensayos inéditos

Doi de artículo: 10.17533/udea.unipluri.19.2.03

Cómo citar este artículo:

Sánchez-Barbero, B; Calatayud, M., y Chamoso, J. M. (2019). Análisis de la interacción de maestros cuando resuelven problemas realistas conjuntamente con sus alumnos en aulas de primaria, teniendo en cuenta su experiencia docente. Uni-pluriversidad, 19(2), 40-59. doi: 10.17533/udea.unipluri.19.2.03

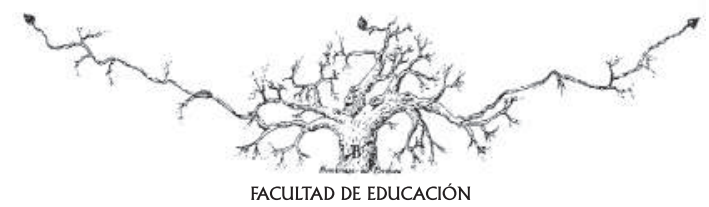

Recibido: 2019-09-14 • Aprobado: 2019-11-14

* Departamento de Didáctica de la Matemática y de las CCEE. Universidad de Salamanca, España. Email: beatrizsanchezb@usal.es

** Departamento de Didáctica de la Matemática y de las CCEE. Universidad de Salamanca, España. Email: idu005769@usal.es

*** Departamento de Didáctica de la Matemática y de las CCEE. Universidad de Salamanca, España. Email: jchamoso@usal.es 


\title{
Resumen
}

La formación de docentes de matemáticas de Primaria necesita conocer y analizar la práctica real del aula. Este trabajo se centra en la resolución conjunta de problemas en las aulas. La investigación educativa ha mostrado que existe escaso razonamiento y participación de los alumnos, cuando maestro y alumnos resuelven de forma conjunta problemas rutinarios en aulas de matemáticas de Primaria. Algunos trabajos muestran que ese razonamiento y participación aumenta cuando resuelven problemas no rutinarios, algo que no siempre ocurre cuando los maestros son noveles. Por ello se pretende analizar qué sucede cuando se utiliza un tipo especial de problemas no rutinarios como los realistas, que la literatura ha considerado con interés, teniendo en cuenta la experiencia del docente. De forma concreta, el objetivo de este trabajo es analizar la interacción entre dos maestras, una experta y una novel, cuando resuelven conjuntamente problemas realistas con sus estudiantes en su aula habitual, atendiendo a los procesos que se promueven, el grado de participación y la experiencia docente de las maestras. Los resultados mostraron que aumentó tanto el razonamiento como la participación de los alumnos, y que la maestra experta promovió más el razonamiento y realizó una mejor interpretación realista de los problemas en la resolución que la novel. Aunque se trata de una muestra escasa, estos resultados permiten dar un paso para abrir futuras líneas de investigación que podrían tener implicaciones educativas, tanto para los docentes en formación como para la formación continua de los que están ejerciendo.

Palabras clave: Interacción en el aula, Educación Matemática, problemas realistas, formación de profesores, Educación Primaria

\begin{abstract}
Training of Primary mathematics teachers needs to know and analyze the real practice of the classroom. This work focuses on a joint resolution of problems in the classroom. Educational research has shown that there is little reasoning and participation of students when teachers and students jointly solve routine problems in Primary math classrooms. Some studies showed that this reasoning and participation increases when they solve non-routine problems, but it does not always happen when teachers are novel. Therefore, it is intended to analyze what happens when a special type of non-routine problems such as realistic problems is used, which the literature has considered with interest, taking into account the experience of the teacher. Specifically, the objective of this work is to analyze the interaction between two teachers, an expert and a novel, when they solve realistic problems along with their students in their usual classroom, attending to the processes, degree of participation, and teaching experience of the teachers. The results showed that both reasoning and participation of the students increased, and the expert teacher promoted the reasoning more than the novel and also, she made a better realistic interpretation of the problems in the resolution. Although it is a small sample, these results allow us to take a step to open future lines of research that could have educational implications for both preservice teachers and in-service teachers.
\end{abstract}

Keywords: Classroom Interaction, Mathematics Education, realistic problems, teacher training, Primary Education. 


\section{INTRODUCCIÓN}

La formación de profesores de matemáticas de primaria necesita analizar la práctica real del aula. Conocer qué sucede cuando los docentes resuelven tareas matemáticas de forma conjunta con los estudiantes en las aulas de Primaria, es un tema que ha suscitado interés en Educación Matemática y que podría ayudar a enriquecer esa formación (por ejemplo, Chapman, 2006; Depaepe, De Corte y Verschaffel, 2010; Rosales, Vicente, Chamoso, Múñez y Orrantia, 2012).

La resolución de problemas es una de las tareas cognitivas más importantes que se desarrolla en las aulas de matemáticas (Kolovou, van den Heuvel-Panhuizen y Bakker, 2009) ya que dota a los alumnos de conocimientos, habilidades y destrezas para afrontar situaciones realistas y problemáticas (Aksoy, Bayazit y Kirnap, 2015). Cai y Lester (2010) afirman que las oportunidades de aprendizaje de los alumnos dependen del tipo de tarea que se desarrolla en las aulas y de cómo interaccionan profesor y alumnos en dicho desarrollo.

Algunos estudios han mostrado que se produce un escaso razonamiento y parti- cipación de los alumnos cuando resuelven problemas rutinarios (por ejemplo, Chapman, 2006; Depaepe et al., 2010; Rosales et al., 2012), donde incluso los resultados numéricos no tienen una lógica aparente (Verschaffel, De Corte y Boghart, 1997). Existen escasos trabajos con problemas no rutinarios, pero en sus resultados aumenta tanto el razonamiento como la participación de los estudiantes (Sánchez, Carrillo, Vicente y Juárez, 2015; Sánchez-Barbero, Chamoso, Vicente y Rosales, 2018; Sánchez-Barbero et al., 2019). Por lo tanto, en este trabajo se pretende utilizar un tipo especial de problemas no rutinarios que la literatura ha considerado con interés, los problemas realistas (por ejemplo, Aksoy et al., 2015; Kolovou et al., 2009; Mellone, Verschaffel y Van Dooren 2017; Verschaffel et al., 1997; Verschaffel y De Corte, 1997), donde la experiencia docente puede influir. En consecuencia, en este trabajo se analiza la interacción que se produce entre dos maestras, una experta y otra novel, y sus estudiantes cuando resuelven de forma conjunta problemas realistas en el aula.

\section{Marco Teórico}

El marco teórico se organiza en dos partes. Por un lado, se refiere a tareas que se puede utilizar para aprender matemáticas y, por el otro, a la interacción que se produce entre docentes, teniendo en cuenta su experiencia, y sus estudiantes cuando resuelven de forma conjunta problemas en el aula.

\subsection{Tareas para aprender matemáticas}

Los problemas que se utilizan para aprender matemáticas tienen un gran impacto en el aprendizaje de los alumnos (Mullins, Martin, Ruddock, O'Sullivan y Preuschoff, 2012). Dependiendo de su nivel de comple- 
jidad ofrecen oportunidades, por ejemplo, para potenciar la reflexión del resolvente y favorecer su aprendizaje matemático (Elbers, 2003). Cada tipo de problema atiende al objetivo que se pretenda conseguir, por lo que se seleccionarán cuidadosamente para favorecer el aprendizaje y la formación completa de la competencia matemática de los estudiantes (Chandia, Rojas, Rojas y Howard, 2016; Zaslavsky, 2007).

Entre las diversas clasificaciones de problemas, en este trabajo se consideran dos tipos: problemas rutinarios, aquellos que para resolverlos basta con la aplicación directa de algoritmos o conocimientos previos, y problemas no rutinarios, aquellos que no tienen un único camino inmediato de resolución, sino que, para llegar a la solución, exigen utilizar estrategias que deben ser propuestas por el resolvente para alcanzar la meta planteada (Jiménez y Verschaffel, 2014). Entre este último tipo de problemas se incluyen los problemas realistas, aquellos que reproducen una situación que los estudiantes pueden proyectar en su mente (Van den Heuvel-Panhuizen, 2003, citado en Beswick, 2011). Para su resolución se necesita utilizar tanto conocimiento matemático como información situacional del problema y, además, razonamiento para decidir la solución adecuada (Mellone et al., 2017; Vicente y Orrantia, 2017).

La resolución de problemas para el aprendizaje matemático es un tema de gran interés en Educación Matemática (Koichu, 2014). A pesar de que la investigación en resolución de problemas se centró durante décadas en aspectos relacionados directamente con el proceso de resolución (Jiménez y Verschaffel, 2014), posteriormente ha tenido otros muchos objetivos, por ejemplo, por un lado, considerar el texto del problema con identidad propia (Gerofsky, 1997) o, por el otro, entender la resolución de problemas como un elemento cultural (Lave, 1992), como un juego con reglas claras e iguales para todas las personas que forman parte de él (Verschaffel y De Corte, 1997) o como un instrumento para favorecer el razonamiento y la participación de los estudiantes para el aprendizaje (Rosales et al., 2012).

\subsection{Interacción en aulas de matemáti- cas}

Considerar la interacción en el aula de matemáticas conlleva referirse al concepto de "enseñanza dialógica" que se basa en la interpretación de hechos que se producen en las aulas entre docente y alumnos; es un recurso creativo para estos últimos, que depende del objetivo que el primero se haya propuesto (Bakker, Smit y Wegerif, 2015). La enseñanza dialógica pretende construir conocimiento a través del diálogo en función de la perspectiva que se adopte (Elbers, 2003) y producir un aprendizaje significativo, para lo que es necesario conectar el nuevo conocimiento con los conocimientos que se poseen (Ausubel, Novak y Hanesian, 1983). Es importante que promueva el aprendizaje por medio del razonamiento y la participación de los alumnos, que ha de ser negociada para conseguirlo (Planas y Morera, 2011).

Puesto que gran parte de los conocimientos que adquiere el alumno se desarrolla en contextos de aula, analizar la interacción entre maestro y alumnos en el aula ayuda a conocer cómo se produce el aprendizaje matemático (Cai y Lester, 2010; Rosales, Orrantia, Vicente y Chamoso, 2008a; 2008b; Rosales et al., 2012). Se quieren utilizar tareas que promuevan interacciones donde el docente no se limite a hablar y los alumnos a responder (Burgos, Domínguez, Rojas, Planas y Vilella, 2006), sino que los alumnos exterioricen ideas justificando sus pensamientos y compartiéndolos con los 
compañeros, construyendo su aprendizaje (Lampert, 1990; Planas y Morera, 2011).

En el presente trabajo se considera un tipo concreto de interacción, la que se realiza entre maestros y alumnos cuando resuelven conjuntamente problemas en el aula de matemáticas de Primaria, un momento importante para favorecer el aprendizaje matemático. En esa interacción se consideran dos aspectos concretos: por un lado, los Procesos que se promueven y, por otro, el Grado de Participación de maestros y alumnos en dicha interacción. En referencia a los Procesos que se promueven, se consideran tanto Procesos Cognitivos, en función del nivel de razonamiento que se produce en la interacción (Praetorius, Pauli, Reusser, Rakoczy y Klieme, 2014; Smart y Marshall, 2013), como Procesos Metacognitivos (Flavell, 1985; 1987), entendidos como la capacidad reflexiva y regulatoria de cualquier actividad, que hace tomar conciencia de la propia cognición (Riveros et al., 2010). Respecto al Grado de Participación de maestros y alumnos en la resolución conjunta de problemas, se entiende como la participación que tienen en la construcción del conocimiento para el aprendizaje.

Estudios que analizan la resolución conjunta de problemas rutinarios entre maestros y alumnos en las aulas de matemáticas de Primaria se han desarrollado en diversos sentidos. Por ejemplo, Chapman (2006) analizó la influencia del contexto social con catorce maestros de Primaria cuando resolvían un problema rutinario y los resultados mostraron que la interacción durante la resolución del problema se reducía a la selección de datos, con escaso razonamiento y poca participación de los alumnos. De forma similar, Depaepe et al. (2010), basándose en Chapman (2006), compararon el comportamiento de dos maestros cuando resolvían problemas rutinarios de libros de texto y los resultados mostraron que los maestros se centraban en la selección de datos numéricos más que en el razonamiento. Rosales et al. (2012) analizaron la interacción de once maestros de Primaria con sus estudiantes frente a problemas rutinarios a los que añadieron un problema reescrito entendido como aquel problema que incluye información matemática y situacional para promover la comprensión del problema (Staub y Reusser, 1995, citado en Vicente y Orrantia, 2007); los resultados corroboraron estudios previos, ya que la interacción en ambos tipos de problemas se basó en la selección y ejecución de algoritmos, con escaso razonamiento y escasa participación de los alumnos (en la línea de otros trabajos previos de los mismos autores como, por ejemplo, Rosales et al.(2008a; 2008b). Nathan y Knuth (2003) analizaron la participación de una maestra y sus alumnos cuando resolvían problemas rutinarios en el aula de matemáticas de Primaria durante dos años consecutivos y los resultados mostraron una escasa participación de los alumnos que crecía según avanzaba el nivel de escolarización (en la línea de González y DeJarnette, 2015).

Se conocen escasos estudios en los que se analizara la interacción con problemas no rutinarios en contextos de Primaria y en condiciones similares al trabajo que se presenta. Por ejemplo, los resultados de Sánchez et al. (2015) reflejaron que se produjo un incremento del interés y participación de los alumnos y del razonamiento. Sánchez-Barbero et al. (2018) analizaron los Procesos Metacognitivos que se producían en la interacción de diez maestros cuando cada uno de ellos resolvía de forma conjunta sus alumnos un problema no rutinario y los resultados mostraron que dichos Procesos aparecían escasamente, en su mayor parte aludiendo a aspectos generales que podían ayudar a la resolución más que a aspectos regulatorios. En otros niveles educativos, Escribano, Sánchez-Barbero y Chamo- 
so (2019) analizaron la interacción que se producía cuando un profesor universitario resolvía conjuntamente diez problemas realistas con sus alumnos, futuros docentes, y los resultados mostraron un aumento del razonamiento en la resolución y una amplia presencia de Procesos Metacognitivos, especialmente de regulación referidos a planificación, supervisión y evaluación; además, los alumnos solicitaban más problemas similares para poder resolverlos.

La literatura también aporta otros trabajos que compararon la resolución conjunta de maestros, expertos y noveles, de problemas rutinarios con sus estudiantes en aulas de matemáticas de Primaria. Los resultados de Blanton, Berenson y Norwood (2001) mostraron cómo los docentes noveles dejaban mayor participación a los alumnos que sus iguales expertos. Chamoso, Vicente, Rosales y Orrantia (2007) señalaron cómo los maestros expertos eran los que permitían mayor autonomía a los alumnos y concluían la resolución de los problemas con una respuesta correcta, algo que no siempre se producía con los maestros noveles y que, además, tampoco era siempre fruto de una resolución conjunta del problema. Rosales et al. (2008a; 2008b) confirmaron que los maestros expertos se centraban más en la promoción del razonamiento, mientras que los maestros noveles se centraban en la selección y ejecución de la operación para resolver los problemas; ambos grupos de maestros facilitaban una escasa autonomía a los alumnos donde los noveles lo hicieron en menor grado. En otros niveles educativos como secundaria, Sánchez-Barbero et al. (2019) mostraron cómo, independientemente de la experiencia docente, tanto docentes expertos como noveles, de forma similar en ambos casos, promovían en gran medida el razonamiento en la resolución de problemas no rutinarios.
En otro sentido, Verschaffel, Greer y De Corte (2000) verificaron cómo los maestros noveles no utilizaban el conocimiento del mundo real cuando resolvían problemas realistas.

La revisión de la literatura permite concluir que, cuando los docentes resuelven problemas rutinarios de forma conjunta con los estudiantes en el aula de Primaria, se promueve escaso razonamiento y existe poca participación de los alumnos. En los escasos estudios similares en los que se utilizaron problemas no rutinarios parece que aumentó el razonamiento y la participación de los estudiantes. Estas razones animan a considerar el análisis de la interacción entre maestro y alumnos cuando resuelven de manera conjunta tipo especial de problemas no rutinarios que la literatura ha considerado interesante, los problemas realistas. El estudio se realiza con una maestra experta y una novel para poder valorar si la experiencia docente influye en la interacción cuando se realiza la resolución. Los resultados pueden facilitar información interesante tanto para la formación de futuros maestros de Primaria como para los que están en activo.

En concreto, el objetivo de este trabajo es comparar la interacción que se produce entre dos maestras, experta y novel, y sus respectivos estudiantes en la resolución conjunta de problemas realistas en aulas de Primaria, teniendo en cuenta los Procesos que se promueven y el Grado de Participación de maestras y alumnos. En este trabajo se entiende por maestro experto aquel que tiene una experiencia docente superior a diez años y como maestro novel aquel que no tiene experiencia docente. La literatura aporta poca información acerca de la influencia de la experiencia docente en el análisis de la interacción conjunta con problemas realistas en el sentido del trabajo que se presenta, pero permite plantear las siguientes hipótesis: 
1. Atendiendo a los Procesos Cognitivos, la maestra experta promueve un mayor grado de razonamiento que la novel.

2. Atendiendo a los Procesos Metacognitivos, la maestra novel promueve una mayor regulación que la experta.

3. Atendiendo al Grado de Participación, la maestra experta cede mayor participación a los alumnos que la no- vel cuando se produce razonamiento, mientras que la novel cede mayor participación a los alumnos en los procesos regulatorios.

4. Atendiendo a la interpretación de los problemas, la maestra experta considera el realismo de los problemas en la resolución en mayor medida que la novel.

\section{Metodología}

Participantes: Dos maestras, una novel (graduada en Maestro de Primaria, que estaba cursando el máster de Estudios Avanzados de Dificultades de Aprendizaje en la Universidad de Salamanca, España) y una experta (con 10 años de experiencia). Las maestras son elegidas por disponibilidad y sus 29 y 27 estudiantes, respectivamente, son de tercer ciclo de Educación Primaria (11-12 años) de un colegio de Sa- lamanca, España. Las maestras informaron que no había ningún alumno con dificultades de aprendizaje en sus aulas, pero sí un alumno con altas capacidades en el aula de la maestra experta; y que ninguna de ellas había recibido formación específica en resolución de problemas.

Materiales: Se utilizaron diez problemas realistas (Verschaffel, De Corte y Lasure, 1994) que se presentan en la Figura 1

P1. Carlos tiene 9 amigos y Jorge tiene 12 amigos. Carlos y Jorge deciden dar juntos una fiesta. Invitan a todos sus amigos y todos los amigos van a la fiesta. ¿Cuántos amigos van a la fiesta?

P2. Esteban ha comprado 4 tablones de 2.5 metros cada uno. ¿Cuántos tablones de 1 metro puede obtener de esos tablones?

P3. ¿Cuál será la temperatura del agua de un recipiente si mezclas un litro de agua a $80^{\circ}$ y un litro de agua a $40^{\circ}$ en él?

P4. 450 soldados deben ser transportados a su lugar de entrenamiento. En cada autobús puede entrar 36 soldados. ¿Cuántos autobuses serán necesarios?

P5. Juan corre los 100 metros en 17 segundos. ¿Cuánto tardará en correr 1 kilómetro?

P6. Roberto y Alicia van a la misma escuela. Roberto vive a 17 kilómetros de la escuela y Alicia a $8 \mathrm{~km}$. ¿A qué distancia vive Roberto de Alicia?

P7. El abuelo da a sus nietos una caja con 18 globos para repartir entre ellos. ¿Cuántos globos le toca a cada uno?

P8. Andrés nació en 1978. Ahora estamos en 1993. ¿Cuántos años tiene?

P9. Un hombre quiere tener una cuerda lo suficientemente larga para unir dos postes separados entre si por 12 metros, pero solo tiene trozos de cuerda de 1.5 metros. ¿Cuántos trozos necesitaría juntar para hacer la cuerda lo suficientemente larga para unir las estacas?

P10. Este recipiente se está llenando con un grifoa ritmo constante. Si el agua tiene una profundidad de $4 \mathrm{~cm}$. tras 10 segundos, ¿cuánta profundidad tendrá después de 30 segundos?

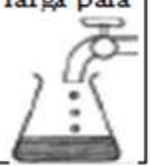

Figura 1. Problemas realistas resueltos conjuntamente por las maestras y sus respectivos estudiantes (traducidos de Verschaffel et al., 1994) 
Procedimiento: Las maestras fueron grabadas en audio mientras resolvían los problemas con sus alumnos en el horario y aula habitual dedicados a realizar este tipo de tareas. Un observador externo realizó anotaciones de aquellos aspectos que no fueron recogidos en el audio. La duración de las grabaciones de la maestra experta y novel fue de 2460 y 2220 segundos, respectivamente. Tanto las maestras como los padres de los estudiantes, por ser menores de edad, firmaron un consentimiento y autorizaron que sus datos se utilizaran para esta investigación.

Análisis: Las grabaciones de las sesiones se transcribieron y analizaron. Para realizar dicho análisis se consideró el ciclo, entendido como la segmentación de las acciones realizadas durante el desarrollo de la interacción en la resolución de una tarea en el aula y que suele comenzar con una pregunta, ya sea de modo explícito o implícito, y finalizar cuando la pregunta ha sido respondida o abandonada (Wells, 1999, citado en Sánchez et al., 2015). Para ello se tuvieron en cuenta los contenidos públicos, entendidos como la información que maestro y alumnos comparten en el aula de forma explícita, de manera que cada ciclo contiene un único contenido público (si en un mismo ciclo existe más de un contenido público, se considera principal aquel del que dependan otro u otros contenidos públicos; Rosales et al., 2012).

Una vez que se delimitaron los ciclos, se clasificaron atendiendo a dos aspectos: los Procesos promovidos en la resolución de los problemas y el Grado de Participación de maestras y alumnos en la interacción.

a. Procesos: (Escribano et al., 2019) Como se muestra en la Tabla 1, se refiere a:

Tabla 1. Sistema de categorías de Procesos (ejemplos extraídos de la experimentación)

\begin{tabular}{|c|c|c|c|}
\hline & CATEGORÍAS & DEFINICIÓN & EJEMPLOS \\
\hline \multirow{2}{*}{ 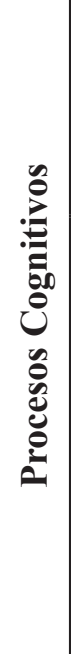 } & Selección & $\begin{array}{l}\text { Aspectos que aparecen explíci- } \\
\text { tamente en el enunciado del pro- } \\
\text { blema o surgen en el proceso de } \\
\text { resolución, sin justificación. }\end{array}$ & $\begin{array}{l}\mathrm{P}: \text { ite ha dado? } \\
\text { A: } 21 . \\
\mathrm{P}: 21 .\end{array}$ \\
\hline & Integración & $\begin{array}{l}\text { Aspectos que relacionan o } \\
\text { comparan información o datos, } \\
\text { que aparecen explícitamente } \\
\text { en el enunciado del problema } \\
\text { o surgen en el proceso de re- } \\
\text { solución, de forma adecuada y } \\
\text { justificada. }\end{array}$ & $\begin{array}{l}\text { P: ¿Entonces qué tendriamos que } \\
\text { saber? } \\
\text { A: Tendríamos que saber dos cosas: } \\
\text { una, si Alicia está a } 8 \mathrm{~km} \text {. de la } \\
\quad \text { escuela o de la casa de Roberto } \\
\text { y a cuántos km. está la escuela } \\
\text { de cada casa. } \\
\text { A: ¡Claro! Eso es lo que pienso yo. }\end{array}$ \\
\hline
\end{tabular}




\begin{tabular}{|c|c|c|c|}
\hline & CATEGORÍAS & DEFINICIÓN & EJEMPLOS \\
\hline \multirow{2}{*}{ 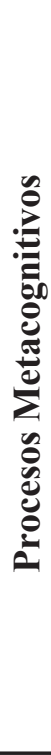 } & Generalización & $\begin{array}{l}\text { Aspectos del proceso de reso- } \\
\text { lución que son más generales } \\
\text { que los considerados en el pro- } \\
\text { blema. }\end{array}$ & $\begin{array}{l}\text { P: A ver, pensad...pensad un poqui- } \\
\text { to... Pablo, piensa un poquito... } \\
\text { Tenemos que mezclar un litro } \\
\text { de agua a } 80^{\circ} \text { y un litro de agua } \\
\text { a } 40^{\circ} \text {. Vale, ¿y ahora se pone a } \\
120^{\circ} \text { ? ¿Hierve el agua cuando } \\
\text { le pones agua más fría? }\end{array}$ \\
\hline & Regulación & $\begin{array}{l}\text { Aspectos del proceso de resolu- } \\
\text { ción relacionados con acciones } \\
\text { de planificación (organización } \\
\text { del proceso), supervisión (valo- } \\
\text { ración y observación del proce- } \\
\text { so) y evaluación (determinación } \\
\text { del avance y progreso en la re- } \\
\text { solución, así como valoración } \\
\text { de la realización del proceso). }\end{array}$ & $\begin{array}{l}\text { P: Vale a ver os explico un momenti- } \\
\text { to, vamos, os voy a dar una hoja } \\
\text { con una serie de problemas, } \\
\text { los vais a tener ahi, los vais a } \\
\text { pensar en grupo, ¿vale? Voy a } \\
\text { dejar } 5 \text { minutillos para que los } \\
\text { penséis en grupo, y luego, y lue- } \\
\text { go, los vamos a ir resolviendo } \\
\text { ¿vale?, los vamos a ir resolvien- } \\
\text { do entre todos. }\end{array}$ \\
\hline \multirow{2}{*}{ 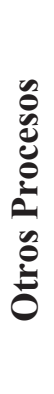 } & Control & $\begin{array}{l}\text { Aspectos de mantenimiento de } \\
\text { la atención y el orden de la cla- } \\
\text { se, u organizativos, sin relación } \\
\text { en ningún sentido con el proce- } \\
\text { so de resolución. }\end{array}$ & $\begin{array}{l}\text { P: No mováis las mesas, por favor. } \\
\quad \text { ¿Podéis tranquilizaros? De ver- } \\
\quad \text { dad... }\end{array}$ \\
\hline & Lectura & $\begin{array}{l}\text { Aspectos de lectura del proble- } \\
\text { ma, que incluye definición de } \\
\text { vocabulario previo al proceso } \\
\text { de resolución. }\end{array}$ & $\begin{array}{l}\text { A: (...) ¿Cuántos trozos necesitaría } \\
\text { juntar para hacer la cuerda lo } \\
\text { suficientemente larga para unir } \\
\text { las estacas? }\end{array}$ \\
\hline
\end{tabular}

\section{Fuente: Elaboración propia}

Otros Procesos (Control y Lectura, $8.40 \%$ y $14.00 \%$ del total de los ciclos de las maestras, respectivamente) no se consideraron en este trabajo.

b. Grado de Participación de maestras y alumnos (adaptado de Escribano et al., 2019; Rosales et al., 2012; Turner et al., 2012) La tabla 2 indica quién asume la construcción del contenido público del ciclo

Tabla 2. Sistema de categorías de Grado de Participación (ejemplos extraídos de la experimentación)

\begin{tabular}{|c|c|c|c|}
\hline & $\begin{array}{l}\text { GRADO DE } \\
\text { PARTICIPACIÓN }\end{array}$ & INDICADORES & EJEMPLOS \\
\hline 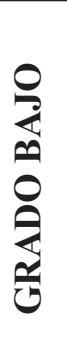 & $\begin{array}{l}\text { Grado } \mathbf{P} \text { (profesor): } \\
\text { El maestro de forma } \\
\text { autónoma }\end{array}$ & $\begin{array}{l}\text { El maestro inicia el ciclo } \\
\text { y lo puede finalizar. }\end{array}$ & $\begin{array}{l}\text { P: Entonces, ¿qué podemos decir } \\
\text { aquí? ¿Cuánto tardará? Depende } \\
\text { de la persona y depende... Y } \\
\text { también de, isssssh! Tardará } \\
\text { mucho más en recorrer, ¿vale? } \\
\text { No es la misma proporción, no es } \\
\text { proporcional el recorrer un km } \\
\text { que el recorrer } 100 \mathrm{~m} \text {. }\end{array}$ \\
\hline
\end{tabular}




\begin{tabular}{|c|c|c|c|}
\hline \multicolumn{2}{|r|}{$\begin{array}{c}\text { GRADO DE } \\
\text { PARTICIPACIÓN }\end{array}$} & INDICADORES & EJEMPLOS \\
\hline 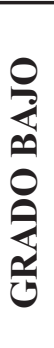 & $\begin{array}{l}\text { Grado Pa } \\
\text { (profesor-alumnos): } \\
\text { El maestro y alumno, } \\
\text { con una mayor } \\
\text { participación del } \\
\text { maestro }\end{array}$ & $\begin{array}{l}\text { - El maestro inicia el ciclo } \\
\text { con una pregunta cerrada } \\
\text { o invasiva que puede in- } \\
\text { cluir parte de la respuesta. } \\
\text { - El maestro puede finali- } \\
\text { zar el ciclo con un feed- } \\
\text { back. }\end{array}$ & $\begin{array}{l}\text { P: Yo corro lo mismo } 100 \text { metros } \\
\text { que } 1 \text { km. Vale, venga. Corro } \\
\text { igual de rápido los } 100 \text { metros } \\
\text { que hay en un kilómetro a } 100 \\
\text { metros solos... } \\
\text { A: No. }\end{array}$ \\
\hline \multirow[t]{2}{*}{ 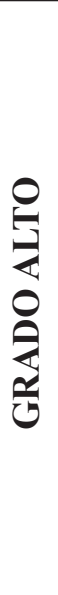 } & $\begin{array}{l}\text { Grado Ap (alumnos- } \\
\text { profesor): El maestro } \\
\text { y alumno, con una } \\
\text { mayor participación del } \\
\text { alumno }\end{array}$ & $\begin{array}{l}\text { - El maestro inicia el ciclo } \\
\text { con una pregunta o inter- } \\
\text { vención abierta que no } \\
\text { incluye parte de la res- } \\
\text { puesta. } \\
\text { - El maestro o alumno } \\
\text { pueden finalizar el ciclo } \\
\text { con un feedback. }\end{array}$ & $\begin{array}{l}\text { P: Álvaro. } \\
\text { A: Es que claro, a ver... Si corres } \\
\text { 100 metros y luego te quedan } \\
\text { otros } 10 \text {, o sea otros } 100 \text { estás } \\
\text { más cansado y otros } 100, \text { y otros } \\
\text { 100, cada vez estás más cansado y } \\
\text { tardas más. } \\
\text { A: O depende... } \\
\text { A: Intenta hacerlo tú. }\end{array}$ \\
\hline & $\begin{array}{l}\text { Grado A (alumnos): } \\
\text { El alumno de forma } \\
\text { autónoma }\end{array}$ & $\begin{array}{l}\text { El alumnado inicia el ci- } \\
\text { clo y lo puede finalizar. }\end{array}$ & $\begin{array}{l}\text { A: “Haz al mismo tiempo un } \\
\text { kilómetro al mismo tiempo todo el } \\
\text { rato". }\end{array}$ \\
\hline
\end{tabular}

Fuente: Elaboración propia

En este trabajo se consideraron Grado Bajo, aquellos ciclos en los que el maestro tuvo la mayor participación en la construcción de su idea principal (Grado P y Grado Pa) y Grado Alto, aquellos ciclos en los que el alumno tuvo mayor participación en la construcción de su idea principal (Grado Ap y Grado A). Posteriormente se contabilizó el número de ciclos, en valor absoluto y en porcentajes, atendiendo a los Procesos y al Grado de Participación de la maestra novel y la maestra experta, y se realizó un análisis cuantitativo. Para este análisis se utilizó el estadístico Chi-cuadrado $(\alpha=.05)$ para descubrir si existían diferencias significativas entre los Procesos que se promovieron y el Grado de Participación, cuando cada maestra y sus alumnos resolvieron conjuntamente problemas realistas.

a. Fiabilidad: Dos investigadores (dos autores de este trabajo) analizaron la totalidad de los ciclos de forma independiente y compararon sus resultados (índice de Kappa de Cohen de: ciclos, 0.99; Procesos, 0.89 y Grado de Participación, 0.95). Los desacuerdos se resolvieron con discusión y posterior consenso entre ambos.

b. Medidas: Hace referencia a los siguientes aspectos:

a) Procesos promovidos: se comparó el porcentaje medio de ciclos dedicados, por un lado, a los Procesos Cognitivos (Selección e Integración) y, por otro, a los Procesos Metacognitivos (Generalización y Regulación).

b) Grado de Participación: se comparó el porcentaje medio de ciclos dedicados al Grado Bajo y Grado Alto de maestras y alumnos. 
c) Procesos y Grado de Participación: se comparó el porcentaje de ciclos de Procesos Cognitivos y de Procesos Metacognitivos con relación a los Grados de Participación. d) Interpretación del realismo de los problemas en la resolución: se comparó si las maestras consideraban el realismo de los problemas para decidir la solución.

\section{Resultados}

Los resultados del análisis de la interacción de dos maestras, experta y novel, mientras resolvían con sus alumnos problemas realistas en aulas de Primaria muestran que, globalmente, en relación con los Procesos, más de dos tercios de los ciclos, 68.93\%, fueron dirigidos a la promoción de Procesos Cognitivos, mientras que el $31.07 \%$ se dirigió a Procesos Metacognitivos. Si se analizan los resultados teniendo en cuenta la experiencia docente, la maestra experta promovió mayor porcentaje de Procesos Cognitivos en la interacción que la novel (novel, $65.00 \%$ vs. experta, $74.42 \%$ ) y, por tanto, la maestra novel dedicó un porcentaje mayor a los Procesos Metacognitivos que la maestra experta $(35.00 \%$ frente al $25.58 \%$, respectivamente).

Tabla 3. Porcentajes medios globales de los ciclos

\begin{tabular}{|c|c|r|r|r|}
\cline { 3 - 5 } \multicolumn{2}{|c}{} & EXPERTA & \multicolumn{1}{c|}{ NOVEL } & \multicolumn{1}{c|}{ GLOBAL } \\
\hline \multirow{3}{*}{ Procesos cognitivos } & Selección & $23.26 \%$ & $26.67 \%$ & $25.24 \%$ \\
\cline { 2 - 5 } & Integración & $51.16 \%$ & $38.33 \%$ & $43.69 \%$ \\
\hline \multirow{2}{*}{ Procesos metacognitivos } & Regulación & $13.95 \%$ & $33.33 \%$ & $25.24 \%$ \\
\cline { 2 - 5 } & Generalización & $11.63 \%$ & $1.67 \%$ & $5.83 \%$ \\
\hline
\end{tabular}

Fuente: Elaboración propia

Centrándose en los Procesos Cognitivos, los resultados globales muestran que en casi dos tercios de los ciclos se promovió la Integración, $63.38 \%$, y en el $36.62 \%$ la Selección. Al considerar la experiencia docente de las maestras, el comportamiento de ambas fue similar, sin diferencias significativas (Selección, $\mathrm{p}=.239$; Integración, $\mathrm{p}=.377$ ), con un predominio del proceso de Integración tanto en la maestra novel como en la experta (Figura 2), resultado que contradice la hipótesis 1.Con relación a los Procesos Metacognitivos, los resultados globales muestran que la mayor parte de los ciclos promovieron la Regulación, $81.25 \%$, mientras que la promoción de la Generalización fue de $18.75 \%$. Teniendo en cuenta la experiencia docent e de las maestras, estas se comportaron de forma diferente en la resolución de problemas realistas, con diferencias significativas (Regulación, $\mathrm{p}=.0001$; Generalización, $\mathrm{p}=.000$ ). Existe un predominio del proceso de Regulación en la maestra novel, mientras que la maestra experta mantuvo una promoción equitativa de Regulación y Generalización (Figura 2), resultados que apoyan la hipótesis 2 . 


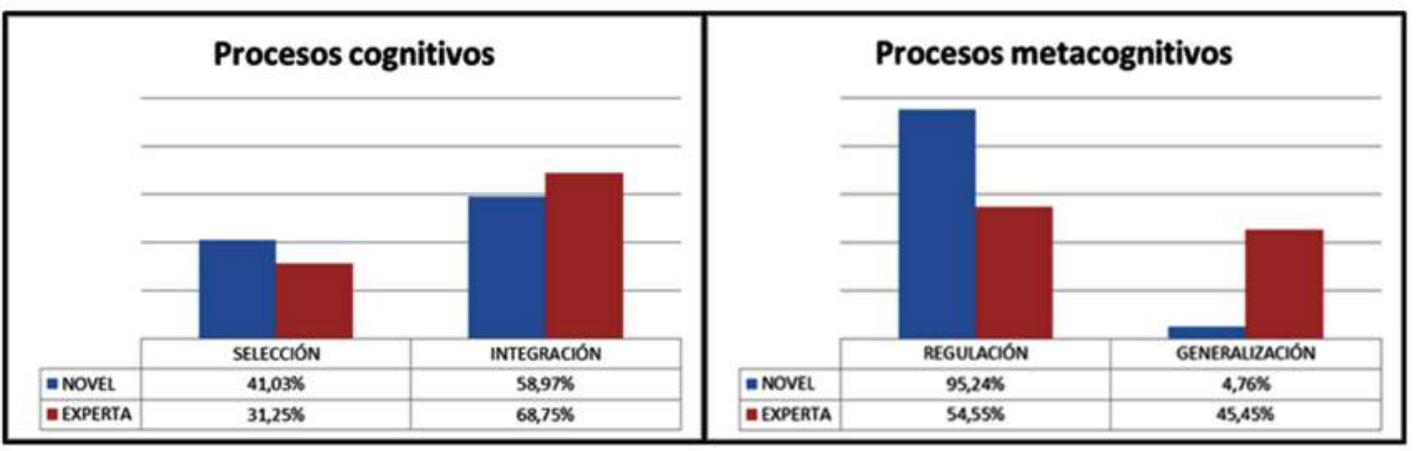

Fuente: Elaboración propia

Figura 2. Porcentaje medio de ciclos dedicados por las maestras a los Procesos Cognitivos y Metacognitivos

En relación con el Grado de Participación, los resultados globales muestran que aproximadamente la mitad de los ciclos tenían un Grado Bajo de participación y la otra mitad un Grado Alto. Teniendo en cuenta la experiencia docente, la participación de las dos maestras y sus alumnos se repartió casi equitativamente, aunque la maestra novel cedió mayor autonomía a los alumnos, $54.17 \%$, frente al $44.44 \%$ de la maestra experta, sin diferencias significativas (Figura 3; Grado Bajo, p=.424; Grado Alto, $\mathrm{p}=.230$ ).

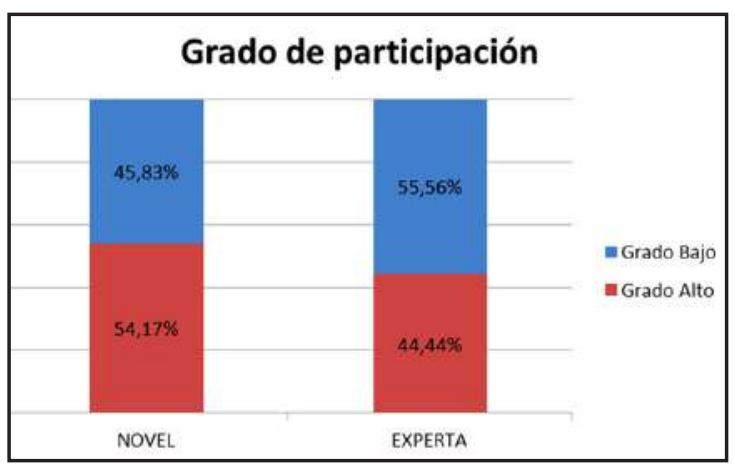

Fuente: Elaboración propia

Figura 3. Porce ntaje medio de ciclos dedicados por las maestras al Grado de Participación
Si se analiza el Grado de Participación teniendo en cuenta los Procesos (Figura 4), los resultados globales muestran que los Procesos Cognitivos promovieron un Grado Alto de participación (Selección, 61.54\%; Integración, 70.00\%), mientras que los Procesos Metacognitivos lo hicieron en Grado Bajo (Regulación, 86.54\%; Generalización, $75.00 \%)$. Teniendo en cuenta la experiencia docente, los resultados muestran cómo la maestra experta mantuvo prácticamente igualado el Grado Bajo y el Grado Alto de participación tanto en Selección como en Integración, mientras que la maestra novel dejó mayor autonomía a los alumnos en ambos Procesos Cognitivos, con más del $80 \%$ en Integración, y con diferencias significativas entre ambas (Selección, $\mathrm{p}=.007$; Integración, $\mathrm{p}=.000$ ). Referido al Grado de Participación teniendo en cuenta los Procesos Metacognitivos, los resultados muestran cómo ambas maestras tuvieron un alto porcentaje de Grado Bajo en Regulación, sin diferencias significativas ( $\mathrm{p}=.065)$, y que en Generalización la maestra novel tuvo una participación equitativa en el aula, mientras que la experta cedió menor participación a los alumnos, con diferencias significativas $(\mathrm{p}=.000)$. Estos resultados contradicen la hipótesis 3 . 


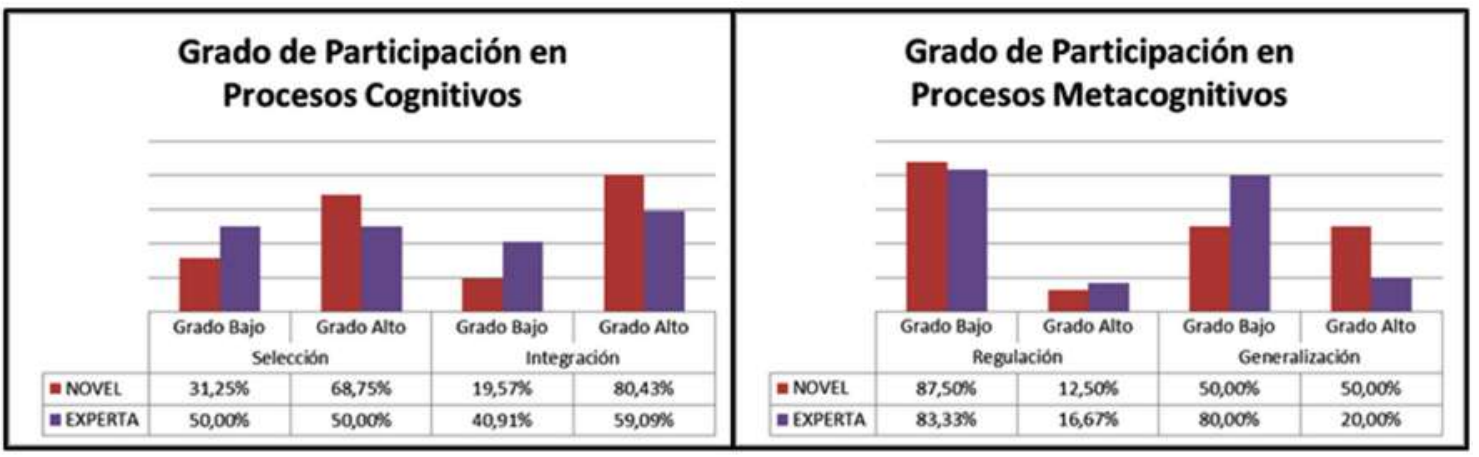

Fuente: Elaboración propia

Figura 4. Porcentaje medio de ciclos dedicados por las maestras al Grado de Participación teniendo en cuenta los Procesos Cognitivos y Metacognitivos

Referido a la interpretación del realismo de los problemas, la maestra experta lo hizo con todos ellos, mientras que la maestra novel no lo hizo con los problemas 2, 3, 5 y 8 (Tabla 4). Estos resultados apoyan la hipótesis 4.

Tabla 4. Interpretación realista de los problemas por cada maestra

\begin{tabular}{|l|c|c|}
\cline { 2 - 3 } \multicolumn{1}{c|}{} & \multicolumn{2}{c|}{ Interpretación realista } \\
\cline { 2 - 3 } \multicolumn{1}{c|}{} & Experta & Novel \\
\hline Problema 1 & SÍ & SÍ \\
\hline Problema 2 & SÍ & NO \\
\hline
\end{tabular}

\begin{tabular}{|l|c|c|}
\cline { 2 - 3 } \multicolumn{1}{c|}{} & \multicolumn{2}{c|}{ Interpretación realista } \\
\cline { 2 - 3 } \multicolumn{1}{c|}{} & Experta & Novel \\
\hline Problema 3 & SÍ & NO \\
\hline Problema 4 & SÍ & SÍ \\
\hline Problema 5 & SÍ & NO \\
\hline Problema 6 & SÍ & SÍ \\
\hline Problema 7 & SÍ & SÍ \\
\hline Problema 8 & SÍ & NO \\
\hline Problema 9 & SÍ & SÍ \\
\hline Problema 10 & SÍ & SÍ \\
\hline
\end{tabular}

Fuente: Elaboración propia

\section{Discusión}

Este estudio analiza si la experiencia docente implica un cambio en la interacción cuando maestros resuelven de manera conjunta problemas realistas con sus alumnos en aulas de Primaria, atendiendo a los Procesos que se promueven, al Grado de Participación de maestros y alumnos, y a la Interpretación realista de estos problemas en dicha resolución. Se esperaba que, en general, el tipo de problemas influyera en que se produjera una mayor Integración que Selección. Además, se esperaba que la maestra experta promoviera en mayor medida la Integración y la Generalización que la maestra novel, que cediera mayor Grado de Participación a sus alumnos en dichos procesos y que diera mayor Interpretación realista a la resolución de los problemas.

Con respecto a los Procesos Cognitivos, las maestras promovieron principalmente el razonamiento (mayor porcentaje de Integración que de Selección), resultados que difieren de los de otros trabajos similares con problemas rutinarios (Chamoso et al., 2007; Chapman, 2006; Depaepe et al., 2010; Rosales et al., 2008a; 2008b; Rosales et al., 2012), y son cercanos a otros en que se uti- 
lizaron problemas no rutinarios (Sánchez et al., 2015; Sánchez-Barbero et al., 2019). El tipo de problema utilizado, que puede suponer un reto para el alumno, influyó en el razonamiento que se produjo en la interacción, aunque se necesitaría más investigación sobre ello. Además, la Integración estuvo más presente en la interacción de la maestra experta, quizás debido al llamado enriquecimiento que se produce con la experiencia, fruto del contacto cotidiano tanto con los problemas que se resuelven en el aula, como con la forma de hacerlo y la manera en que los alumnos se comportan en ese proceso (aunque sean problemas de los libros de texto, usualmente típicos problemas rutinarios; Sánchez-Barbero et al., 2018). Sin embargo, no existen evidencias para afirmar que la experiencia docente influyera en la promoción del tipo de Procesos que se produjeron en la interacción.

Con respecto a los Procesos Metacognitivos, las maestras promovieron aspectos regulatorios (mayor porcentaje de Regulación que de Generalización) en mayor proporción que cuando se utilizaron problemas rutinarios en aulas de Primaria, donde la promoción de la Generalización era prácticamente inexistente (Sánchez-Barbero et al., 2018) y más cercanos a los de Sánchez-Barbero et al. (2019), donde destacó un alto porcentaje de procesos de Regulación cuando los problemas eran no rutinarios. Además, los resultados mostraron que el comportamiento de ambas maestras fue diferente, ya que la maestra novel promovió la Regulación y apenas la Generalización, mientras que la maestra experta lo hizo de forma similar en ambos aspectos. El alto porcentaje de Regulación de la maestra novel pudo deberse a que, por su escasa experiencia docente, intentara desarrollar la sesión de manera secuenciada y ordenada (Lepage et al., 2005).
Por el contrario, la experiencia de la maestra experta pudo favorecer en mayor medida la Generalización, que lleva implícita una contextualización del problema o de su resolución atendiendo a aspectos más generales que los del mismo problema que pueden ayudar a su desarrollo. En este aspecto pudo influir que los problemas fueran realistas, aunque la escasez de trabajos en este sentido hace que se aconseje más investigación sobre ello.

Con respecto al Grado de Participación, los resultados globales mostraron que la participación de maestras y alumnos fue equitativa, resultados que difieren de los obtenidos en otros trabajos similares con problemas rutinarios, donde los alumnos tenían escasa participación en la interacción (Blanton et al., 2001; Chamoso et al., 2007; Rosales et al., 2008a; 2008b), y son más cercanos a trabajos que utilizaron problemas no rutinarios (Sánchez-Barbero et al., 2018; Sánchez-Barbero et al., 2019). Estos resultados pueden hacernos pensar que el tipo de tarea que se utiliza en el aula influye en la participación de los estudiantes si esas tareas hacen que se cuestionen su conocimiento e ideas, en la línea de trabajos como Carrillo, Climent, Gorgorió, Prat y Rojas (2008) y Sánchez et al. (2015).

En el Grado de Participación en los Procesos Cognitivos, los resultados globales mostraron un Grado Alto tanto en Selección como en Integración, resultados que difieren de los de Blanton et al. (2001), Chamoso et al. (2007) y Rosales et al. (2008a; 2008b), donde los alumnos tenían escasa participación en la promoción del razonamiento, y están en la línea con Sánchez-Barbero et al. (2019) en otros niveles educativos. Teniendo en cuenta la experiencia del docente, se observó cómo la maestra novel participó más que los alumnos en la construcción de 
ideas en los ciclos de Selección y menos en los de Integración, mientras que la maestra experta lo hizo equitativamente en ambos aspectos. Atendiendo al Grado de Participación en relación con los Procesos Metacognitivos, estos fueron llevados por las dos maestras, resultados que son similares a los de Sánchez-Barbero et al. (2018).

Con respecto a la Interpretación del realismo de los problemas en la resolución, la maestra experta lo hizo con la totalidad de los problemas, mientras que la novel sólo en el $60 \%$. Esto pudo deberse a que maestra experta tenía un alumno con altas capacidades y tenía gran conocimiento del alumnado y de sus posibilidades al llevar trabajando juntos casi dos años. Estos resultados se asemejan a los de otros estudios como el de Verschaffel et al. (2000), en el que se evidenció que los maestros en formación mostraron una fuerte tendencia a no utilizar el conocimiento del mundo real y no realizar consideraciones realistas al resolver problemas; o el de Kilic (2017), donde los alumnos resolvían problemas realistas con su maestro novel ignorando esa interpretación realista de los mismos, aunque más investigación sobre ello sería necesaria.

\section{Conclusiones}

En este trabajo se analizó la influencia de la experiencia docente en la resolución conjunta de problemas realistas que se genera entre docentes y sus estudiantes en el aula de Primaria, atendiendo a los Procesos que se promovieron, al Grado de Participación de docentes y alumnos, y a la Interpretación realista de los problemas en la resolución.

En primer lugar, se considera que las herramientas utilizadas permitieron analizar de manera minuciosa los Procesos y el Grado de Participación cuando maestros y alumnos resuelven conjuntamente tareas en el aula, basadas en la literatura y fruto de un proceso de depuración en diversos estudios previos (Rosales et al., 2008a; 2008b; Rosales et al.,2012) que se continúan con este trabajo. Los resultados permiten confiar en dichas herramientas para una futura investigación. Puesto que estas han sido utilizadas para analizar la interacción que se produce en el aula entre docente y alumnos en la resolución de diferentes tipos de problemas, también podrían utilizarse como elemento de formación docente, tanto para maestros en formación como para los que están en ejercicio, al permitir mostrar aspectos que se consideran fundamentales para favorecer el aprendizaje matemático, como son el fomento del razonamiento y de la participación de los alumnos. Además, esas herramientas podrían adaptarse para analizar la interacción que se produce entre maestros y alumnos en el aula cuando trabajan en otras áreas de conocimiento.

En segundo lugar, a partir de los resultados del estudio, se podría decir que, respecto a los Procesos Cognitivos promovidos, globalmente existió una mayor promoción del razonamiento que en otros trabajos con problemas rutinarios, lo que hizo una interacción más interesante (Burgos et al., 2006), donde ambas maestras se comportaron de manera similar. Por otra parte, respecto a los Procesos Metacognitivos, globalmente existió una mayor promoción de la Regulación con estrategias que implicaban una planificación, supervisión y evaluación de los da- 
tos; el comportamiento de ambas maestras fue diferente, pues la novel promovió mayoritariamente la Regulación, mientras que la experta favoreció principalmente la Generalización. Esta conclusión puede abrir futuras líneas de investigación.

En tercer lugar, respecto al Grado de Participación de maestras y alumnos en la interacción, fue similar en ambos casos. Relacionando ese Grado de Participación con los Procesos Cognitivos, globalmente existió un Grado Alto de participación. Además, tanto en el aula de la maestra experta como en la de la novel se promocionó en mayor medida la Integración que coincidió con una alta participación de los alumnos. La maestra novel fue la que más delegó la construcción de la idea principal de los ciclos en sus alumnos. En Selección, la maestra novel también favoreció la autonomía de los alumnos en la construcción de la idea principal de los ciclos, mientras que la maestra experta limitó esa autonomía a sus alumnos. Si se establece la relación del Grado de Participación con los Procesos Metacognitivos, existió de forma global un Grado Bajo de participación. Las maestras se comportaron en ambas aulas de forma parecida en cuanto a que fueron las responsables de la construcción de las ideas principales de ciclos de Regulación, aunque no en los de Generalización, donde la maestra experta restó autonomía a los alumnos y, en cambio, en el aula de la maestra novel, alumnos y maestra fueron protagonistas de la construcción de ideas de forma similar.

En cuarto lugar, respecto a la interpretación de la resolución, la maestra experta interpretó de manera realista la totalidad de los problemas con los que se trabajaron, mientras que la maestra novel interpretó de este modo el $60 \%$ de los problemas. Aunque estos resultados requieren mayor investiga- ción, podrían sugerir la importancia de introducir problemas de este tipo en el contexto de formación de docentes.

Es importante conocer la práctica de aula, aunque es difícil conseguir que los maestros permitan que se recoja su actuación en ella. Este trabajo permite valorarlo en un estudio de casos de maestros experto y novel. A pesar de lo reducido de la muestra, sus resultados pueden tener implicaciones educativas en cuanto a la conveniencia de introducir problemas realistas para la formación y desarrollo docente. Además, permite abrir futuras líneas de investigación en diferentes sentidos como, por ejemplo, ampliar la muestra para comparar resultados teniendo en cuenta peculiaridades de los docentes, los alumnos y el contexto, y el tipo de tarea que se utiliza para resolver problemas. También se puede analizar los ciclos comenzados por los alumnos y estudiar hacia qué proceso se dirigen. Otra posibilidad se relaciona con profundizar en las causas del diferente tratamiento que las maestras experta y novel hicieron del realismo de los problemas en la resolución. Otra vía de investigación sería analizar la interacción que se produce en el aula entre docentes y alumnos con otros problemas no rutinarios, como los problemas abiertos o en aulas donde se utilizan diferentes metodologías como el Aprendizaje Basado en Proyectos. También se podría analizar la interacción que se produce en aulas con estudiantes con dificultades de aprendizaje, al resolver problemas de forma conjunta con el docente. Ampliar el estudio a otras etapas educativas como Secundaria o Universidad u otras áreas de conocimiento cuando se trabajan tareas no rutinarias, podría ser otra posibilidad.

Los resultados parecen indicar que debería trabajarse en las aulas con tareas no rutinarias, diferentes a las propuestas en 
los libros de texto, que tienden a ser mecánicas (Depaepe et al., 2010; Vicente, Rosales, Chamoso y Múñez, 2013) y en las que la promoción del razonamiento y la dotación de autonomía al alumno suele quedar en manos del docente (Kolovou et al., 2009). De esta forma se promovería el razonamiento en las aulas de matemáticas de manera que los alumnos sean capaces de relacionar la práctica de aula con su vida cotidiana, y se favorezca la autonomía de los alumnos. Esta acción tendría implicaciones para el profesorado, que precisaría una formación que favorezca su práctica docente relacionada con la comprensión y resolución de tareas no rutinarias (Boston, 2013; Sullivan et al., 2015).

\section{REFERENCIAS BIBLIOGRÁFICAS}

Aksoy, Y., Bayazit, I., y Kirnap, S. M. (2015). Prospective Primary school teachers' proficiencies in solving real-words problems: approaches, strategies and models. Eurasia Journal of Mathematics, Science \& Technology Education, 1(4), 827-839.

Ausubel, D., Novak, J., y Hanesian, H. (1983). Psicología Educativa: Un punto de vista cognoscitivo. México: Trillas.

Bakker, A., Smit, J., y Wegerif, R. (2015). Scaffolding and dialogic teaching in mathematics education: introduction and review. ZDM Mathematics Education, 47, 1047-1065.doi: 10.1007/s11858015-0738-8.

Beswick, K. (2011). Putting context in context: an examination of the evidence for the benefits of “contextualized" tasks. International Journal of Science and Mathematics Education 9(2), 367390. doi:10.1007/s10763-011-9323-y.

Blanton, M. L., Berenson, S. B., y Norwood, K. (2001). Using classroom discourse to understand a prospective mathematics teacher's developing practice. Teaching and Teacher Education, 17, 227-242.

Boston, M. D. (2013). Connecting changes in secondary mathematics teachers' knowledge to their experiences in a profesional development workshop. Journal of Mathematics Teacher Education, 16(1), 7-31. doi: 10.1007/s10857-012-9211-6.

Burgos, S., Domínguez, M., Rojas, F.J., Planas, N., y Vilella, X. (2006). La participación en el aula de Matemáticas. En J. M. Goñi (coord.), Matemáticas e Interculturalidad (49-62). Barcelona: Graó.

Cai, J., y Lester, F. (2010). Why is teaching with problem solving important to student learning? Reston, VA: National Council of Teachers of Mathematics.

Carrillo, J., Climent, N., Gorgorió, N., Prat, M., y Rojas, F. (2008). Análisis de secuencias de aprendizaje matemático desde la perspectiva de la gestión de la participación. Enseñanza de las Ciencias, 26(1), 67-76.

Chamoso, J. M., Vicente, S., Rosales, J. y Orrantia, J. (2007). Análisis de la interacción profesor-alumno en el aula de matemáticas: actividades, autonomía e incidentes. En E. Mancera \& C. A. Pérez, Historia y Prospectiva de la Educación Matemática. Memorias de la XII CIAEM (págs. 77-97). Méjico: Edebé Internacional. 
Chandia, E., Rojas, D., Rojas, F. y Howard, S. (2016). Creencias de formadores de profesores de matemáticas sobre resolución de problemas. Bolema, Rio Claro (SP), 30, 605 - 624. doi: 10.1590/1980-4415v30n55a15.

Chapman, O. (2006). Classroom practices for context of mathematics Word problems. Educational Studies in Mathematics, 62(2), 211-230. doi: 10.1007/s10649-006-7834-1.

Depaepe, F., De Corte, E. y Verschaffel, L. (2010). Teachers' approaches towards Word problem solving: elaborating or restricting the problem context. Teaching and Teacher Education, 26(2), 152-160.

Elbers, E. (2003). Classroom interaction as reflection: learning and teaching mathematics in a community of inquiri. Educational Studies in Mathematics, 54, 77-99.

Escribano, M., Sánchez-Barbero, B. y Chamoso, J. M. (2019). Análisis de la interacción profesor-alumnos cuando resuelven problemas realistas en el aula. Memorias de la XIII CIAEM.

Flavell, J.H. (1985). Cognitive Development. New York: Prentice-Hall

Flavell, J.H. (1987). Speculations about the nature and development of metacognition. En: F. E. Weinert \& R. H. Kluwe (Eds). Metacognition, Motivation and Understanding. Hillsdale, NJ: Erlbaum.

Gerofsky, S. (1997). A linguistic and narrative view of word problems in mathematics education. For the Learning of Mathematics, 16(2), 36-45.

González, G. y DeJarnette, A.F. (2015). Teachers' and students' negotiation moves when teachers scaffold group work. Cognition and Instruction, 33(1), 1-45.

Jiménez, L. y Verschaffel, L. (2014). Development of Children's solutions of non-standard arithmetic word problem solving. Revista de Psicodidáctica, 19(1), 93-123.

Kiliç, Ç. (2017). The Realistic Reasons for Unrealistic Solutions of Pre-Service Primary School Mathematics Teachers in Non-Standard Word Problems: The Example from Turkey. Mustafa Kemal University Journal of Social Sciences Institute, 14(37), 285-298.

Koichu, B. (2014) (with contributions by G. Goldin, I. Weinzweig, S. Vinner and R. Leikin). Reflections on problem solving. En M. N. Fried \& T. Dreyfus (Eds.), Mathematics \& Mathematics Education: Searching for Common Ground. Advances in Mathematics Education (pp. 113-135). Dordrecht, The Netherlands: Springer.

Kolovou, A., van den Heuvel-Panhuizen, M. y Bakker, A. (2009). Non-routine problem solving tasks in Primary School Mathematics textbooks-a needle in a Haystack. Mediterranean Journal for Research in Mathematics Education, 8(2), 31-68.

Lampert, M. (1990). When the problem is not the question and the solution is not the answer: Mathematical knowing and teaching. American Educational Research Journal, 27, 29-63.

Lave, J. (1992). Word problems: A microcosm of theories of learning. Context and Cognition: Ways of Learning and Knowing, 74-92. New York, NY: Harvester Wheatsheaf. 
Lepage, P., Darling-Hammond, L. Akar, H. Gutierrez, C. Jenkins-Gunn, E., y Rosebrock, K. (2005). Classroom Management. En L. Darling-Hammond y J. Bransford (Eds.), Preparing teachers for a changing world, what teachers should learn and able to do (pp.327-357). San Francisco: Jossey-Bass.

Mellone, M., Verschaffel, L. y Van Dooren, W. (2017). The effect of rewording and dyadic interaction on realistic reasoning in solving Word problems. The Journal of Mathematical Behavior, 46, $1-12$.

Mullins, I., Martin, M., Ruddock, G., O'Sullivan, C. y Preuschoff, C. (2012). TIMSS 2011. Marcos de la Evaluación. Madrid: Ministerio de Educación, Cultura y Deporte.

Nathan, M.J. y Knuth, E.J. (2003). A study of who classroom mathematical discourse and teacher change. Cognition and Instruction, 21(2), 175-207.

Planas, N. y Morera, L. (2011). Educación Matemática e interacción en el aula de secundaria. Uno. Revista de Didáctica de las Matemáticas, 58, 77-83.

Praetorius, A. K., Pauli, C., Reusser, K., Rakoczy, K. y Klieme, E. (2014). One lesson is all you need? Stability of instructional quality across lessons. Learning and Instruction, 31, 2-12.

Riveros, M., Zanocco, P., Cnudde, V., Espinoza, F., Rojas, X. y Baeza, P. (2010). Habilidades de pensamiento metacognitivo y resolución de problemas matemáticos. Boletín de Investigación Educacional, 15(1), 89-107. Facultad de Educación, Pontificia Universidad Católica de Chile, Santiago, Chile.

Rosales, J., Orrantia, J., Vicente, S. y Chamoso, J. M. (2008a). La resolución de problemas aritméticos en el aula. ¿Qué hacen los profesores cuando trabajan conjuntamente con sus alumnos? Cultura y Educación, 20(4), 423-439.

Rosales, J., Orrantia, J., Vicente, S. y Chamoso, J. M. (2008b). Studying mathematics problem-solving classrooms. A comparision between the discourse of in-service teachers and student teachers. European Journal of Psychology of Education, 23(3), 275-294.

Rosales, J., Vicente, S., Chamoso, J. M., Muñez, D. y Orrantia, J. (2012). Teacher- student interaction in joint word problem solving. The role of situational and mathematical knowledge in mainstream classrooms. Teaching and Teacher Education, 28(8), 1185-1195.

Sánchez, B., Carrillo, J., Vicente, S. y Juárez, J. A. (2015). Análisis de la interacción alumnos-profesor al resolver problemas no rutinarios en aulas de Primaria. En XIV Conferencia interamericana de Educación Matemática (XIV CIAEM). Chiapas (México).

Sánchez-Barbero, B., Chamoso, J. M., Vicente, S. y Rosales, J. (2018). Análisis de la metacognición en la interacción profesor-alumnos al resolver problemas de matemáticas en aulas de Primaria. En L. J. Rodríguez-Muñiz, L. Muñiz-Rodríguez, A. Aguilar-González, P. Alonso, F. J. García García y A. Bruno (Eds.), Investigación en Educación Matemática XXII (pp. 544-553). Gijón: SEIEM.

Sánchez-Barbero, B., Ciudad, J. C., Galán, E., Chamoso, J. M., Vicente, S., Rodríguez, M. M., Cáceres, M. J. y Salomón, M. S. (2019). Análisis de la interacción profesor-alumnos cuando resuelven 
conjuntamente problemas realistas en aulas de Secundaria. En J. M. Marbán, M. Arce, A. Maroto, J. M. Muñoz-Escolano y Á. Alsina (Eds.), Investigación en Educación Matemática XXIII (p. 650). Valladolid: SEIEM.

Sullivan, P., Askew, M., Cheeseman, J., Clarke, D., Mornane, A., Roche, A. y Walker, N. (2015). Supporting teachers in structuring mathematics lessons involving challenging tasks. Journal of Mathematics Teacher Education, 18(2), 123-140.

Smart, J. B. y Marshall, J. C. (2013). Interactions between classroom discourse, teacher questioning, and student cognitive engagement in middle school science. Journal of Science Teacher Education, 24(2), 249-267.

Staub, F. C. y Reusser, K. (1995). The role of presentational structures in understanding and solving mathematical word problems. En C. A. Weaver III, S. Mannes \& C. R. Fletcher (Eds.), Discourse Comprehension: Essays in honor of Walter Kintsch (pp. 285-305). Hillsdale, NJ: Lawrence Erlbaum.

Turner, J. C., Midgley, C., Meyer, D. K., Gheen, M., Anderman, E. M., Kang, Y. y Patrick, H. (2002). The classroom environment and students' reports of avoidance strategies in mathematics: A multimethod study. Journal of Educational Psychology, 94(1), 88.

Van den Heuvel-Panhuizen, M. (2003). The didactical use of models in realistic mathematics education: An example from a longitudinal trajectory on percentage. Educational Studies in Mathematics, 54, 9-35.

Verschaffel, L., De Corte, E. y Borghart, I. (1997). Pre-service teachers' conceptions and beliefs about the role of real-world knowledge in mathematical modelling of school word problems. Learning and Instruction, 7, 339-359.

Verschaffel, L., De Corte, E. y Lasure, S. (1994). Realistic considerations in mathematical modeling of school arithmetic word problems. Learning and Instruction, 4, 273-294.

Verschaffel, L. Greer, B. y De Corte, E. (2000). Making sense of word problems. Lisse: Swets \& Zeitlinger.

Vicente, S. y Orrantia, J. (2007). Resolución de problemas y comprensión situacional. Cultura y Educación, 19(1), 61-85.

Vicente, S., Rosales, J., Chamoso, J. M. y Múñez, D. (2013). Análisis de la práctica educativa en clases de matemáticas españolas de Educación Primaria: Una posible explicación para el nivel de competencia de los alumnos. Cultura y Educación, 25(4), 535-548.

Wells, G. (1999). Dialogic inquiry: Toward a sociocultural practice and theory of education. Cambridge: CUP.

Zaslavsky, O. (2007). Mathematics-related task, teacher education, and teacher educator. Journal of Mathematics Teacher Education, Londres, 10(4-6), 433-440. doi: 10.1590/1980-4415v30n55a15. 